Pacific

Journal of

Mathematics

\title{
HOW LARGE ARE THE SPECTRAL GAPS?
}

Alex Iosevich and Steen Pedersen 


\section{HOW LARGE ARE THE SPECTRAL GAPS?}

\section{Alex Iosevich And Steen Pedersen}

Let $D$ be a bounded domain in $\mathbb{R}^{n}$ whose boundary has a Minkowski dimension $\alpha<n$. Suppose that $E_{\Lambda}=\left\{e^{2 \pi i x \cdot \lambda}\right\}_{\lambda \in \Lambda}$, $\Lambda$ an infinite discrete subset of $\mathbb{R}^{n}$, is a frame of exponentials for $L^{2}(D)$, with frame constants $A, B, A \leq B$. Then if

$$
R \geq C\left(\frac{B|\partial D|_{\alpha}}{A|D|}\right)^{\frac{1}{n-\alpha}},
$$

where $C$ depends only on the ambient dimension $n$ and $|\partial D|_{\alpha}$ denotes the Minkowski content, then every cube of sidelength $R$ contains at least one element of $\Lambda$. We give examples that illustrate the extent to which our estimates are sharp.

Let $D$ be a domain of finite Lebesgue measure in $\mathbb{R}^{n}$. Suppose that $L^{2}(D)$ has a frame of exponentials of the form $E_{\Lambda}=\left\{e^{2 \pi i x \cdot \lambda}\right\}, \lambda \in \Lambda$, a discrete infinite subset of $\mathbb{R}^{n}$, with frame constants $A, B, A \leq B$, in the sense that

$$
A\|f\|_{L^{2}(D)}^{2} \leq \sum_{\Lambda}|\hat{f}(\lambda)|^{2} \leq B\|f\|_{L^{2}(D)}^{2},
$$

where $f: D \rightarrow \mathbb{C}$, and $\hat{f}$ denotes the standard Fourier transform. In this paper we will work with frames rather than exponential basis because $L^{2}$ of every bounded domain has frames, whereas exponential basis are generally hard to come by. (See $[\mathbf{F u g}]$.) The following quantities were introduced by Beurling. See $[\mathbf{B r}]$.

$$
D_{R}^{+}=\max _{x \in \mathbb{R}^{n}} \#\left\{\Lambda \cap Q_{R}(x)\right\},
$$

where $Q_{R}(x)$ is a cube of sidelength $2 R$ centered at $x$, and let

$$
D_{R}^{-}=\min _{x \in \mathbb{R}^{n}} \#\left\{\Lambda \cap Q_{R}(x)\right\} .
$$

It follows from results proved by Landau ([Lan], see also $[\mathbf{G R}])$ that if $D$ is a bounded domain then

$$
\limsup _{R \rightarrow \infty} \frac{D_{R}^{-}}{(2 R)^{n}} \geq|D| .
$$

If the set $E_{\Lambda}$ is actually an orthogonal basis for $L^{2}(D)$ then the inequality (3) is actually an equality for both $D_{R}^{-}$and $D_{R}^{+}$. These results show that, 
asymptotically, a sufficiently large cube centered at any point contains the number of elements of $\Lambda$ at least equal to its volume multiplied by the Lebesgue measure of $D$. In this paper we will show that if the Minkowski dimension, $\alpha$, of the boundary $\partial D$ is smaller than the ambient dimension $n$, then there exists

$$
R=C\left(\frac{B|\partial D|_{\alpha}}{A|D|}\right)^{\frac{1}{n-\alpha}}
$$

where $C$ only depends on $n$ and $|\partial D|_{\alpha}=\varlimsup_{\epsilon \downarrow 0} \epsilon^{\alpha-n}|\{x: d(x, \partial D)<\epsilon\}|$ denotes the $\alpha$-dimensional upper Minkowski content of $\partial D$, such that a cube of sidelength $2 R$ centered at any point contains at least one element of $\Lambda$. Note that if $\partial D$ is, say, piecewise smooth, then $\alpha=n-1$ and $R=C \frac{B|\partial D|}{A|D|}$.

A note on notation. The letter $C$ below shall denote a generic constant which may change from line to line. We shall give more precise information about the constants when appropriate.

Our main result is the following.

Theorem 1. Let $D$ denote a domain in $\mathbb{R}^{n}$ with finite non-zero Lebesgue measure whose boundary $\partial D$ has Minkowski dimension $\alpha<n$ in the sense that

$$
\left|\left\{x \in \mathbb{R}^{n}: d(x, \partial D)<\epsilon\right\}\right| \leq C \epsilon^{n-\alpha} .
$$

Then there exists $C$ depending only on $n$, such that if

$$
R \geq C\left(\frac{B|\partial D|_{\alpha}}{A|D|}\right)^{\frac{1}{n-\alpha}}
$$

then

$$
\Lambda \cap Q_{R}(\mu) \neq \emptyset
$$

for every $\mu \in \mathbb{R}^{n}$, and any set $\Lambda$ such that $E_{\Lambda}$ is an exponential frame for $L^{2}(D)$, with frame constants $A, B, A \leq B$ where $Q_{R}(\mu)$ denotes the cube of sidelength $2 R$ centered at $\mu$.

In other words, our result shows, at least if $A=B$, that if $D$ has a fixed volume, then the maximum gap between the elements of $\Lambda$ is no larger than a fixed constant times the the Minkowski content of the boundary. Moreover, the rate of increase depends on the Minkowski dimension of $\partial D$. This idea is illustrated by the following simple example.

Example 2. Let $D=\left[0, a_{1}\right] \times\left[0, a_{2}\right] \times \cdots \times\left[0, a_{n}\right], a_{1} \geq a_{2} \geq \cdots \geq a_{n}>0$, $\Pi_{j=1}^{n} a_{j}=1$. We can take $\Lambda=\prod_{j=1}^{n} \frac{1}{a_{j}} \mathbb{Z}$. It is not hard to see that the largest cube that does not intersect $\Lambda$ has sidelength $2 R=\frac{1}{a_{n}}$. The measure of $\partial D$ 
is $2 \sum_{j=1}^{n} \frac{1}{a_{j}}$. It follows that

$$
\frac{1}{4 n} \leq \frac{R}{|\partial D|} \leq \frac{1}{4}
$$

so $R$ grows linearly with $|\partial D|$.

Example 3. We now spice up the above example to illustrate the fractal phenomenon. Let $D$ be a domain constructed by taking a square $[0,1]^{2}$ and replacing the upper and lower segments by identical fractal curves of Minkowski dimension $1<\alpha<2$. It is not hard to see that $\Lambda$ may be taken to be $\mathbb{Z}^{2}$. (See $[\mathbf{F u g}]$.) We now blow up the domain by the factor of $t>1$ (i.e., we apply the matrix $t I$, where $I$ is the identity matrix). Let $t D$ denote the image of $D$ under that mapping. The set $\Lambda$ must now be taken to be $\left(\frac{1}{t} \mathbb{Z}\right)^{2}$, which tells us that $R$ in Theorem 1 should be $\approx \frac{1}{t}$. On the other hand, $|\partial t D|_{\alpha} \approx t^{\alpha}$, and $|t D|=t^{2}$, so Theorem 1 gives us $R \approx\left(\frac{t^{\alpha}}{t^{2}}\right)^{\frac{1}{2-\alpha}}=\frac{1}{t}$.

The following example shows that if the Lebesgue measure $|D|=0$ the conclusion of Theorem 1 no longer holds.

Example 4. Let $D \subset[0,1]$ denote the Cantor type set consisting of numbers that do not have 1 or 3 in their base 4 expansion. Let $m$ denote the unique probability measure supported on $D$ (see $[\mathbf{F a l}]$ ) given by the equation

$$
\int f(t) d m(t)=\frac{1}{2} \int f\left(\frac{t}{4}\right) d m(t)+\frac{1}{2} \int f\left(\frac{t+2}{4}\right) d m(t) .
$$

One can check that

$$
\widehat{m}(t)=e^{\pi i \frac{2}{3} t} \Pi_{j=0}^{\infty} \cos \left(\frac{\pi t}{2 \cdot 4^{n}}\right) .
$$

If $\Lambda$ is the set of non-negative integers whose base 4 expansion does not contain 2 or 3 , then $E_{\Lambda}$ is an orthonormal basis of $L^{2}(m)$. (See $[\mathbf{J P}]$.)

In particular this shows that the conclusion of Theorem 1 fails miserably in this case.

Example 5. In this example we shall see that there exist families of domains with piecewise smooth boundaries such that the volume of each domain is 1 , the length of the boundary tends to infinity, but $R$, in the sense of Theorem 1, may always be taken to be $\frac{1}{2}+\epsilon$, for any $\epsilon>0$.

Let $D_{k}$ denote the unit square in $\mathbb{R}^{2}$ where the upper and lower edges are replaced by a sawtooth function with $k$ teeth where the height of each tooth is $\frac{1}{2}$. The length $\left|\partial D_{k}\right|$ goes to infinity as $k \rightarrow \infty$. The set $\Lambda$ for each $D_{k}$ is $\mathbb{Z}^{2}$, so $R$, in the sense of Theorem 1 , may always be taken to be $\frac{1}{2}+\epsilon$, for any $\epsilon>0$. This says that the inequality (6) does not sharply describe the behavior of $R$ in this case. However, the proof of Theorem 1 (see the discussion at the end of the proof of Theorem 1 below) shows that in some 
cases $R$ may be taken to be $C \frac{\text { diameter }(D)}{|D|}$, where $C$ depends only on $n$. We shall see that the example given in this paragraph falls into that category.

In all the previous examples we used frames which were actually orthogonal bases. However, this phenomenon persists in the cases when orthogonal exponential basis do not exist and we have to make do with frames.

Example 6. Let $B_{r}$ denote the disc of radius $r$ in $\mathbb{R}^{2}$ centered at the origin. It was shown in [JP2] that $\Lambda=\frac{1}{2 r} \mathbb{Z}^{2}$ is frame for $L^{2}\left(B_{r}\right)$ with constants $A=B=4 r^{2}$. Note that we do not have orthogonal basis becuase, in particular, that would imply that $A=B=\left|B_{r}\right|=\pi r^{2}$. It is well known that $B_{r}$ does not have orthogonal basis of exponentials. See [Fug].

It is clear that $R$, in the sense of Theorem 1 must be taken to be greater than $\frac{1}{4 r}$, which is exactly what Theorem 1 predicts.

The key estimate (see Lemma 9 below) involved in the proof of Theorem 1 is

$$
\sum_{\lambda \notin Q_{R}(\mu)}\left|\widehat{\chi}_{D}(\lambda-\mu)\right|^{2} \leq C \frac{|\partial D|_{\alpha}}{R^{n-\alpha}},
$$

for any $\mu \in \mathbb{R}^{n}$, where $C$ depends only on the dimension and on the frame constant $B$.

This estimate is similar to the estimate that comes up in the theory of irregularities of distributions, (see [Mgr, p. 110]), namely that for any domain $S$ whose boundary is a piecewise $C^{1}$ curve $\mathcal{C}$

$$
\int_{|t| \geq R}\left|\widehat{\chi}_{S}(t)\right|^{2} d t \leq \frac{|\mathcal{C}|}{2 \pi^{2} R}
$$

In fact, our proof of the estimate (11) given in Lemma 9 below uses an idea from the proof of the estimate (12) given by Brandolini, Colzani, and Travaglini in [BCT].

The proof of Theorem 1 is based on the following sequence of lemmae.

Lemma 7. For any $f \in L^{2}(D)$ define

$$
F_{D} f(\xi)=\int_{D} e^{-2 \pi i x \cdot \xi} f(x) d x
$$

and let $\hat{f}$ denote the standard Fourier transform

$$
\hat{f}(\xi)=\int_{\mathbb{R}^{n}} e^{-2 \pi i x \cdot \xi} f(x) d x .
$$

Let $t_{h} f(x)=f(x+h)$, and let $\chi_{D}$ denote the characteristic function of $D$. Then

$$
\begin{aligned}
F_{D} t_{h} \chi_{D}(\lambda) & =e^{2 \pi i \lambda \cdot h} \widehat{\chi}_{D \cap D+h}(\lambda), \\
F_{D} t_{-h} \chi_{D}(\lambda) & =\widehat{\chi}_{D \cap D+h}(\lambda),
\end{aligned}
$$


and

$$
F_{D} \chi_{D}(\lambda)=\widehat{\chi}_{D}(\lambda) \text {. }
$$

The proof is straightforward.

Lemma 8. Let $D$ be as above. Then

$$
\int_{D}\left|\chi_{D}(x+h)-\chi_{D}(x-h)\right|^{2} d x \leq C|h|^{n-\alpha},
$$

and

$$
\int_{D}\left|\chi_{D}(x)-\chi_{D}(x-h)\right|^{2} d x \leq C|h|^{n-\alpha},
$$

with $C \leq C^{\prime}|\partial D|_{\alpha}$, where $C^{\prime}$ depends only on $n$.

Remark. We note again that even though the estimate $C \leq C^{\prime}|\partial D|_{\alpha}$ is best possible over all $h$ 's, for special choices of $h$, the estimate is frequently much better. (See Example 5 above.)

To prove (19) note that the left hand side equals $\mid\{D-(D+h)\} \cup\{(D+$ $h)-D\}\left.|\leq|\left\{x \in \mathbb{R}^{n}: d(x, \partial D)<h\right\}|\leq C| \partial D\right|_{\alpha}|h|^{n-\alpha}$. The proof of (18) is similar.

The key lemma is the following. (See [BCT] for a similar argument.)

Lemma 9. Let $D$ be as above and let $\Lambda$ be such that $E_{\Lambda}$ is a frame of $L^{2}(D)$ with frame constants $A$ and $B, A \leq B$. Then

$$
\sum_{\left\{\lambda \in Q_{2^{k+1}}-Q_{2^{k}}\right\}}\left|\widehat{\chi}_{D}(\lambda)\right|^{2} \leq C B 2^{-k(n-\alpha)},
$$

where $Q_{R}=Q_{R}(0, \ldots, 0)$, and $C$ as in Lemma 8 .

To prove Lemma 9 chose $N$ boxes $A_{k}^{j}$ and $N$ vectors $h_{j}$ such that $2^{-k} \leq$ $\left|h_{j}\right| \leq 2^{-k+1}, \cup_{j=1}^{N} A_{k}^{j}=Q_{2^{k+1}}-Q_{2^{k}}$, and

$$
\left|e^{2 \pi i \lambda \cdot h_{j}}-1\right| \geq 1, \quad \lambda \in A_{k}^{j} \text {. }
$$

Clearly this can be done in any dimension $n$, for a sufficiently large $N=$ $N(n)$.

Now, by triangle inequality

$$
\begin{aligned}
& \left(\sum_{A_{k}^{j}}\left|\widehat{\chi}_{D}(\lambda)\right|^{2}\right)^{\frac{1}{2}} \\
& \leq\left(\sum_{A_{k}^{j}}\left|\widehat{\chi}_{D \cap D+h_{j}}(\lambda)\right|^{2}\right)^{\frac{1}{2}}+\left(\sum_{A_{k}^{j}}\left|\widehat{\chi}_{D}(\lambda)-\widehat{\chi}_{D \cap D+h_{j}}(\lambda)\right|^{2}\right)^{\frac{1}{2}}
\end{aligned}
$$




$$
=I+I I .
$$

By Lemma 7, the frame property, and Lemma 8 we get

$$
\begin{aligned}
I I^{2} & \leq \sum_{\Lambda}\left|\widehat{\chi}_{D}(\lambda)-\widehat{\chi}_{D \cap D+h_{j}}(\lambda)\right|^{2} \\
& =\sum_{\Lambda}\left|F_{D} \chi_{D}(\lambda)-F_{D} t_{-h_{j}} \chi_{D}(\lambda)\right|^{2} \\
& \leq B \int_{D}\left|\chi_{D}(x)-\chi_{D}\left(x-h_{j}\right)\right|^{2} d x \\
& \leq C B\left|h_{j}\right|^{n-\alpha} \leq C B 2^{-k(n-\alpha)} .
\end{aligned}
$$

On the other hand, by (21), Lemma 7, the frame property, and Lemma 8 we get

$$
\begin{aligned}
I^{2} & \leq \sum_{A_{k}^{j}}\left|\widehat{\chi}_{D \cap D+h_{j}}(\lambda)\right|^{2}\left|e^{2 \pi i \lambda \cdot h}-1\right|^{2} \\
& \leq \sum_{\Lambda}\left|\widehat{\chi}_{D \cap D+h_{j}}(\lambda)\right|^{2}\left|e^{2 \pi i \lambda \cdot h_{j}}-1\right|^{2} \\
& =\sum_{\Lambda}\left|F_{D} t_{h_{j}} \chi_{D}(\lambda)-F_{D} t_{-h_{j}} \chi_{D}(\lambda)\right|^{2} \\
& \leq B \int_{D}\left|\chi_{D}\left(x+h_{j}\right)-\chi_{D}\left(x-h_{j}\right)\right|^{2} d x \\
& \leq C B\left|h_{j}\right|^{n-\alpha} \leq C B 2^{-k(n-\alpha)} .
\end{aligned}
$$

\section{Proof of Theorem 1.}

Since $E_{\Lambda}$ is a frame for $L^{2}(D)$ if and only if $E_{\Lambda-\mu}$ is also a frame for $L^{2}(D)$ (with the same frame constants) for any $\mu \in \mathbb{R}^{n}$, and our estimates do not depend on the choice of $\Lambda$, it is sufficient to consider the case $\mu=(0, \ldots, 0)$.

By the frame property and Lemma 7 we get

$$
\begin{aligned}
A|D| \leq \sum_{\Lambda}\left|F_{D} \chi_{D}(\lambda)\right|^{2} & =\sum_{\Lambda}\left|\widehat{\chi}_{D}(\lambda)\right|^{2} \\
& =\sum_{Q_{R}}\left|\widehat{\chi}_{D}(\lambda)\right|^{2}+\sum_{\left\{\lambda \notin Q_{R}\right\}}\left|\widehat{\chi}_{D}(\lambda)\right|^{2} .
\end{aligned}
$$

Using Lemma 9 we see that if $R=2^{k_{0}}$,

$$
\sum_{\left\{\lambda \notin Q_{R}\right\}}\left|\widehat{\chi}_{D}(\lambda)\right|^{2}=\sum_{k=k_{0}}^{\infty} \sum_{Q_{2^{k+1}}-Q_{2^{k}}}\left|\widehat{\chi}_{D}(\lambda)\right|^{2} \leq C B 2^{-k_{0}(n-\alpha)}=\frac{B C}{R^{n-\alpha}}
$$


So by (25) and (26)

$$
\sum_{Q_{R}}\left|\widehat{\chi}_{D}(\lambda)\right|^{2} \geq A|D|-\frac{B C}{R^{n-\alpha}}
$$

which proves that if $R>\left(\frac{B C}{A|D|}\right)^{\frac{1}{n-\alpha}}$, then

$$
\Lambda \cap Q_{R} \neq \emptyset \text {. }
$$

Moreover, the above proof shows that $C \leq C^{\prime}|\partial D|_{\alpha}$ where $C^{\prime}$ depends only on $n$.

Remark. In the proof above the key estimate is $|\{D \cap D-h\}| \leq$ $C|h|^{n-\alpha}|\partial D|_{\alpha}$. While this is the best possible estimate uniform in $h$, in the proof we are have a wide choice of $h$ 's as long as $|h|=2^{-k}$ and the estimates (18), (19), and (21) are satisfied.

This observation can be used to handle the family of examples given by Example 5 above. For convenience we take $\Lambda=\left(\frac{1}{2}, 0\right)+\mathbb{Z}^{2}$. We can now take all $h$ 's in the proof of Theorem 1 of the form $h=\left(h_{1}, 0\right)$ and for this choice of $h$ 's it is easy to check that $\left|\left\{D_{k} \cap D_{k}-h\right\}\right| \leq C|h|$ diameter $\left(D_{k}\right)$, where $C$ is a uniform constant, since the "teeth" of $D_{k}$ 's point in the $y$-direction. Since diameter $\left(D_{k}\right)$ is uniformly bounded above and below, the lack of sharpness of Theorem 1 exposed in Example 5 is resolved for this family of examples.

\section{References}

[Br] A. Beurling, Local harmonic analysis with some applications to differential operators, Some recent advances in the basic sciences, Academic press, 1 (1966).

[BCT] L. Brandolini, L. Colzani and G. Travaglini, Average decay of Fourier transforms and integer points in polyhedra, Ark. Mat., 35 (1997), 253-275.

[Fal] K. Falconer, The geometry of fractal sets, Cambridge University Press, 1986.

[Fug] B. Fuglede, Commuting self-adjoint partial differential operators and a group theoretic problem, J. Funct. Anal., 16 (1974), 101-121.

[GR] K. Gröchenig and H. Razafinjatovo, On Landau's necessary density conditions for sampling and interpolation of band-limited functions, J. London. Math. Soc., $\mathbf{5 4}$ (1996), 557-565.

[IP] A. Iosevich and S. Pedersen, Spectral and tiling properties of the unit cube, Internat. Math. Res. Notices, 16 (1998), 819-828.

[JP] P.E.T. Jorgensen and S. Pedersen, Dense analytic subspaces in fractal $L^{2}$-spaces, J. Anal. Math., 75 (1998), 185-228.

[JP2] Local harmonic analysis for domains in $\mathbb{R}^{n}$ of finite measure, Analysis and Topology, Eds. C. Andreian Cazacu, O. Lehto and Th. M. Rassias, World Scientific Publishing Co., 1997.

[LRW] J. Lagarias, J. Reed and Y. Wang, Orthonormal bases of exponentials for the $n$ cube, 1998, preprint. 
[Lan] H. Landau, Necessary density conditions for sampling and interpolation of certain entire functions, Acta Math., 117 (1967), 37-52.

[Mgr] H. Montgomery, Ten lectures on the interface between analytic number theory and harmonic analysis, CBMS Regional conference series in mathematics, 1994.

Received June 25, 1998. This research was supported in part by the NSF grant DMS9706825 .

Wright State University

DAYTON OH 45435

E-mail address: iosevich@zara.math.wright.edu

Wright State University

DAYTON OH 45435

E-mail address: steen@math.wright.edu

Department of Mathematics

Georgetown University

WASHINGTON, DC 20057 